\title{
DIRAC COHOMOLOGY AND THE BOTTOM LAYER $K$-TYPES
}

\author{
PAVle PANDŽIĆ \\ University of Zagreb, Croatia
}

\begin{abstract}
Let $G$ be a connected real reductive Lie group with a maximal compact subgroup $K$ corresponding to a Cartan involution $\Theta$ of $G$. Let $\mathfrak{q}=\mathfrak{l} \oplus \mathfrak{u}$ be a $\theta$-stable parabolic subalgebra of the complexified Lie algebra $\mathfrak{g}$ of $G$, where $\theta=d \Theta$. Let $L$ be the centralizer of $\mathfrak{q}$ in $G$. We show that, under certain dominance assumptions, cohomological induction with respect to $\mathfrak{q}$ takes irreducible unitary $(\mathfrak{l}, L \cap K)$-modules with nonzero Dirac cohomology to irreducible unitary $(\mathfrak{g}, K)$-modules which also have nonzero Dirac cohomology.
\end{abstract}

\section{INTRODUCTION}

Let $G$ be a connected real reductive Lie group with a Cartan involution $\Theta$, such that the group $K=G^{\Theta}$ of fixed points of $\Theta$ is a maximal compact subgroup of $G$. In $[\mathrm{P}]$, Parthasarathy introduced a Dirac operator $D$ acting on sections of homogeneous spin bundles on $G / K$, and used it successfully to construct the discrete series representations of $G$. The nice properties of $D$ include a nice expression for the "spin Laplacean" $D^{2}$. Parthasarathy's Dirac inequality asserts that for a unitary Harish-Chandra module $M$, one has $D^{2} \geq 0$. This can be translated into an inequality involving the infinitesimal character of $M$ and the $K$-types that appear in $M$. This inequality was crucial in several classification results, like the classification of unitary highest weight modules $([\mathrm{EHW}])$ and the classification of unitary modules with nonzero $(\mathfrak{g}, K)$-cohomology $([\mathrm{VZ}])$.

2010 Mathematics Subject Classification. 22E47.

Key words and phrases. Reductive Lie group, unitary representation, Harish-Chandra module, Dirac operator, Dirac cohomology, cohomological induction, bottom layer.

The research of the author is partially supported by a grant from the Ministry of Science, Education and Sports of Republic of Croatia. 
In [V2], Vogan studied an algebraic version of $D$ and introduced the concept of Dirac cohomology of a Harish-Chandra module $M$, as the quotient of $\operatorname{Ker} D$ by $\operatorname{Im} D \cap \operatorname{Ker} D$ (see the next section for more details). If $M$ is unitary, this is precisely the space of solutions to $D^{2}=0$. So unitary modules with nonzero Dirac cohomology just barely satisfy the Dirac inequality, hence they are in some sense extremal.

The unitary modules with nonzero Dirac cohomology form an interesting class of unitary modules. For example, they include the discrete series, and most of the more general so called $A_{\mathfrak{q}}(\lambda)$ modules. In particular, all modules with nonzero $(\mathfrak{g}, K)$-cohomology also have nonzero Dirac cohomology, and the two notions are in fact related (see [HP1] and [HKP]). Other unitary modules with nonzero Dirac cohomology include all unitary highest weight modules, for which the Dirac cohomology is the same as $\mathfrak{p}^{+}$-cohomology (see $[\mathrm{HPR}]$ ), and some of the unipotent representations (work in progress with Barbasch).

It is therefore an interesting problem to classify all unitary modules with nonzero Dirac cohomology. This note provides a step towards the solution of this problem. Namely, it shows that under certain (rather strong) dominance assumptions, cohomological induction with respect to a $\theta$-stable parabolic subalgebra $\mathfrak{q}$ of $\mathfrak{g}$ takes unitary modules for the Levi subgroup with nonzero Dirac cohomology to unitary modules for $G$ with nonzero Dirac cohomology. The main idea is to use the bottom layer $K$-types. Since these do not always exist, we need to put dominance assumptions on our module. Further assumptions are made to make the analysis of certain tensor products easier. We will also assume that the infinitesimal character of our modules is real, i.e., in the real span of roots. Since we are interested only in representations with nonzero Dirac cohomology, this assumption is not restrictive in view of Theorem 2.1 below.

Our main result partially overlaps with the results of $[\mathrm{HKP}]$, where much more is proved, with weaker assumptions, but only in the case of $A_{\mathfrak{q}}(\lambda) \bmod$ ules. We hope that the results of this note can be extended by using a translation principle for Dirac cohomology, which we hope to develop in future.

In Section 2 we give a brief review of the definition and main properties of Dirac cohomology. In Section 3 we give a brief overview of the definition and basic properties of cohomological induction. Finally in Section 4 we prove the main result, Theorem 4.1 .

\section{Dirac COHOMOlogy}

Let us first quickly review the setting. More details can be found for example in [HP2]. Other good sources for Clifford algebras and spinors are for example $[\mathrm{C}],[\mathrm{K} 1]$ and $[\mathrm{W}]$.

Let $G$ be a connected real reductive Lie group with a Cartan involution $\Theta$ such that the group $K=G^{\Theta}$ of fixed points of $\Theta$ is a maximal compact 
subgroup of $G$. We denote the Lie algebras of $G$ and $K$ by $\mathfrak{g}_{0}$ respectively $\mathfrak{k}_{0}$, and their complexifications by $\mathfrak{g}$ respectively $\mathfrak{k}$. The Cartan decompositions of $\mathfrak{g}_{0}$ and $\mathfrak{g}$ with respect to $\theta=d \Theta$ are

$$
\mathfrak{g}_{0}=\mathfrak{k}_{0} \oplus \mathfrak{p}_{0} ; \quad \mathfrak{g}=\mathfrak{k} \oplus \mathfrak{p} .
$$

These decompositions are orthogonal with respect to any non-degenerate invariant symmetric bilinear form $B$ on $\mathfrak{g}_{0}$ and $\mathfrak{g}$. We fix such a form in the following.

Let $U(\mathfrak{g})$ be the universal enveloping algebra of $\mathfrak{g}$ and let $C(\mathfrak{p})$ be the Clifford algebra of $\mathfrak{p}$ with respect to $B$. Let $b_{1}, \ldots, b_{p}$ be any basis of $\mathfrak{p}$ and let $d_{1}, \ldots, d_{p}$ be the dual basis with respect to $B$. In other words,

$$
B\left(b_{i}, d_{j}\right)=\delta_{i j}, \quad i, j=1, \ldots, p .
$$

The Dirac operator is

$$
D=\sum_{i=1}^{p} b_{i} \otimes d_{i} \in U(\mathfrak{g}) \otimes C(\mathfrak{p}) .
$$

It is an easy exercise to see that $D$ is independent of the basis $b_{i}$, and $K$ invariant for the adjoint $K$-action on $U(\mathfrak{g}) \otimes C(\mathfrak{p})$ in both factors. This Dirac operator was first introduced in $[\mathrm{P}]$ in the analytic setting of spin bundles on $G / K$. For the algebraic setting, see [V2], [HP1] and [K2].

If $M$ is a $(\mathfrak{g}, K)$-module, and if $S$ is a spin module for $C(\mathfrak{p})$, then $M \otimes S$ is a module for the algebra $U(\mathfrak{g}) \otimes C(\mathfrak{p})$ and for the spin double cover $\tilde{K}$ of $K$. In particular, the Dirac operator $D$ acts, and we can define the Dirac cohomology of $M$ to be the $\tilde{K}$-module

$$
H_{D}(M)=\operatorname{Ker} D / \operatorname{Im} D \cap \operatorname{Ker} D .
$$

In this paper we are only interested in unitary modules $M$. In that case, $H_{D}(M)$ is simply $\operatorname{Ker} D=\operatorname{Ker} D^{2}$. Moreover, let $\mathfrak{h}=\mathfrak{t} \oplus \mathfrak{a}$ be a fundamental Cartan subalgebra of $\mathfrak{g}$, so that $\mathfrak{t}$ is a Cartan subalgebra of $\mathfrak{k}$ and $\mathfrak{a}$ is the centralizer of $\mathfrak{t}$ in $\mathfrak{p}$. We embed $\mathfrak{t}^{*}$ into $\mathfrak{h}^{*}$ by extending functionals on $\mathfrak{t}$ to functionals on $\mathfrak{h}$ which are zero on $\mathfrak{a}$. We fix compatible choices of positive roots for $\mathfrak{g}$ and $\mathfrak{k}$, and we denote by $\rho_{\mathfrak{g}}$, respectively $\rho_{\mathfrak{k}}$, the half sums of positive roots for $\mathfrak{g}$ respectively $\mathfrak{k}$. Then by [HP1, Corollary 2.4 and Proposition 2.6], we see

THEOREM 2.1. Let $M$ be an admissible unitary $(\mathfrak{g}, K)$-module with infinitesimal character $\Lambda \in \mathfrak{h}^{*}$. Then the irreducible $\tilde{K}$-module $E(\tau)$ with highest weight $\tau \in \mathfrak{t}^{*}$ appears in $H_{D}(M)$ if and only if the following conditions hold:

1. $E(\tau)$ appears in the $\tilde{K}$-module $M \otimes S$;

2. $\Lambda$ is conjugate to $\tau+\rho_{\mathfrak{k}}$ by an element of the Weyl group $W_{\mathfrak{g}}$.

It will be important in our calculations that in fact the only way a $\tilde{K}$-type $E(\tau)$ contributing to $H_{D}(M)$ can appear in $M \otimes S$ is as a PRV component in 
the tensor product of a $K$-type $E(\mu)$ of $M$ and a $\tilde{K}$-type $E(\gamma)$ of $S$. Recall that the PRV component ([PRV]) of $E(\mu) \otimes E(\gamma)$ is the component with highest weight conjugate to $\mu+\bar{\gamma}$, where $\bar{\gamma}$ is the lowest weight of $E(\gamma)$. This component always appears in the tensor product with multiplicity one. It can be characterized among the components of the tensor product as the one having the shortest infinitesimal character. See [W, Lemma 9.1.4]. Our assertion follows from this last characterization and Parthasarathy's Dirac inequality $D^{2} \geq 0$. For a more detailed explanation, see [HPP, Proposition $3.2]$.

Since all the lowest weights in $S$ are of the form $-\rho(\mathfrak{n} \cap \mathfrak{p})$ for some $\theta$ stable Borel subalgebra $\mathfrak{b}=\mathfrak{h} \oplus \mathfrak{n}$ containing a fixed Borel subalgebra of $\mathfrak{k}$, we conclude

Corollary 2.2. Keeping the notation and assumptions of Theorem 2.1, assume that $E(\tau)$ appears in $H_{D}(M)$. Then

$$
\tau=w(\mu-\rho(\mathfrak{n} \cap \mathfrak{p}))
$$

for some $\mu$ such that $E(\mu)$ appears in $M$, some choice of $\mathfrak{b}$, and some $w \in W_{\mathfrak{k}}$ making $\mu-\rho(\mathfrak{n} \cap \mathfrak{p})$ dominant.

\section{Cohomological induction}

Let $\mathfrak{q}=\mathfrak{l} \oplus \mathfrak{u}$ be a $\theta$-stable Cartan subalgebra of $\mathfrak{g}$. Let $L$ be the centralizer of $\mathfrak{q}$ in $G$. Then $L$ is a closed connected subgroup of $G$, and we can assume that the maximal compact subgroup of $L$ is $L \cap K$. By cohomological induction one means a collection of functors that construct $(\mathfrak{g}, K)$-modules starting from an $(\mathfrak{l}, L \cap K)$-module $Z$. The module $Z$ is first twisted to the module

$$
Z^{\sharp}=Z \otimes \bigwedge^{\text {top }} \mathfrak{u},
$$

where $\mathfrak{l}$ and $L \cap K$ act on $\bigwedge^{\text {top }} \mathfrak{u}$ by the adjoint action.

Now $Z^{\sharp}$ is viewed as a $(\mathfrak{q}, L \cap K)$ module by letting $\mathfrak{u}$ act trivially, and as a $(\overline{\mathfrak{q}}, L \cap K)$ module by letting $\overline{\mathfrak{u}}$ act trivially. Here the bar refers to conjugation with respect to the real form $\mathfrak{g}_{0}$ of $\mathfrak{g}$, and $\overline{\mathfrak{q}}=\mathfrak{l} \oplus \overline{\mathfrak{u}}$ is the parabolic subalgebra of $\mathfrak{g}$ opposite to $\mathfrak{q}$.

Next one constructs the produced and induced $(\mathfrak{g}, L \cap K)$-modules

$$
\operatorname{pro}\left(Z^{\sharp}\right)=\operatorname{Hom}_{U(\mathfrak{q})}\left(U(\mathfrak{g}), Z^{\sharp}\right)_{L \cap K-\text { finite }} ; \quad \operatorname{ind}\left(Z^{\sharp}\right)=U(\mathfrak{g}) \otimes_{U(\overline{\mathfrak{q}})} Z^{\sharp} .
$$

Here $\mathfrak{g}$ acts on $\operatorname{pro}\left(Z^{\sharp}\right)$ by right multiplication in the first variable of Hom, and on ind $\left(Z^{\sharp}\right)$ by left multiplication in the first factor. The group $L \cap K$ acts both on $\operatorname{pro}\left(Z^{\sharp}\right)$ and on $\operatorname{ind}\left(Z^{\sharp}\right)$ by the adjoint action on $U(\mathfrak{g})$ and by the given action on $Z^{\sharp}$.

Finally, one applies the right derived Zuckerman functors $R^{i} \Gamma$ to $\operatorname{pro}\left(Z^{\sharp}\right)$ to obtain the right cohomologically induced modules

$$
\mathcal{R}^{i}(Z)=R^{i} \Gamma\left(\operatorname{pro}\left(Z^{\sharp}\right)\right)
$$


and the left derived Bernstein functors $L^{i} \Pi$ to $\operatorname{ind}\left(Z^{\sharp}\right)$ to obtain the left cohomologically induced modules

$$
\mathcal{L}_{i}(Z)=L^{i} \Pi\left(\operatorname{ind}\left(Z^{\sharp}\right)\right) .
$$

The Zuckerman functor $\Gamma$ is defined on a $(\mathfrak{g}, L \cap K)$-module $V$ as the largest $\mathfrak{g}$-submodule of $V$ on which the action of $\mathfrak{k}_{0}$ exponentiates to $K$. Alternatively,

$$
\Gamma(V)=(R(K) \otimes V)^{(\mathfrak{k}, L \cap K)},
$$

where $R(K)$ is the space of smooth left and right $K$-finite functions on $K$. The (k, $L \cap K)$-invariants are taken with respect to the tensor product of the left regular action in $R(K)$ and the given action $\pi_{V}$ on $V$. The $K$-action on $\Gamma(V)$ is given by the right regular action in $R(K)$. Finally, to describe the $\mathfrak{g}$-action on $\Gamma(V)$, we first identify $R(K) \otimes V$ with the space $R(K, V)$ of finite-range smooth $V$-valued left and right $K$-finite functions on $K$, via

$$
f \otimes v \longmapsto(k \mapsto f(k) v) .
$$

Then for $X$ in $\mathfrak{g}$ and $F \in R(K, V)^{(\mathfrak{k}, L \cap K)}$,

$$
(X F)(k)=\pi_{V}(\operatorname{Ad}(k) X)(F(k)), \quad k \in K .
$$

For more details, see [HP2, Section 5.4]. See also [KV, Chapter II and Chapter $\mathrm{V}]$, but the definitions there are slightly different.

The Bernstein functor $\Pi$ is defined on a $(\mathfrak{g}, L \cap K)$-module $V$ as

$$
\Pi(V)=(R(K) \otimes V)_{(\mathfrak{k}, L \cap K)},
$$

where we are now taking coinvariants with respect to the same $(\mathfrak{k}, L \cap K)$ action on $R(K) \otimes V$ as above. The $(\mathfrak{g}, K)$-action on $\Pi(V)$ is defined in a similar way as on $\Gamma(V)$.

The following version of the vanishing, irreducibility and unitarity results is not the strongest possible, but it will suffice for our purposes. We can assume that $\mathfrak{l}$ contains our fixed fundamental Cartan subalgebra $\mathfrak{h}$ of $\mathfrak{g}$. We also assume that our positive root system $\Delta^{+}(\mathfrak{g}, \mathfrak{h})$, which was chosen to be compatible with $\Delta^{+}(\mathfrak{k}, \mathfrak{t})$, is also compatible with $\mathfrak{q}$, i.e., that it contains $\Delta(\mathfrak{u})$. We now choose positive roots for $\mathfrak{l}$, again in a compatible way, i.e., we set $\Delta^{+}(\mathfrak{l}, \mathfrak{h})=\Delta(\mathfrak{l}, \mathfrak{h}) \cap \Delta^{+}(\mathfrak{g}, \mathfrak{h})$.

This can all be described in terms of the choice of a $\theta$-stable Borel subalgebra $\mathfrak{b}=\mathfrak{h} \oplus \mathfrak{n}$ of $\mathfrak{g}$ contained in $\mathfrak{q}$. The choice of $\mathfrak{b}$ corresponds to the choice of $\Delta^{+}(\mathfrak{g}, \mathfrak{h})=\Delta(\mathfrak{n})$. Then $\mathfrak{b} \cap \mathfrak{k}=\mathfrak{t} \oplus(\mathfrak{n} \cap \mathfrak{k})$ is a Borel subalgebra of $\mathfrak{k}$ and it corresponds to the choice $\Delta^{+}(\mathfrak{k}, \mathfrak{t})$ in the sense that $\Delta^{+}(\mathfrak{k}, \mathfrak{t})=\Delta(\mathfrak{n} \cap \mathfrak{k})$. In the same way, $\mathfrak{b} \cap \mathfrak{l}=\mathfrak{h} \oplus(\mathfrak{n} \cap \mathfrak{l})$ is a Borel subalgebra of $\mathfrak{l}$ and it corresponds to the choice $\Delta^{+}(\mathfrak{l}, \mathfrak{h})$. We can include in this picture also the pair $(\mathfrak{l} \cap \mathfrak{k}, \mathfrak{t})$. As usual, if $\mathfrak{o} \subset \mathfrak{g}$ is an $\mathfrak{h}$-invariant subspace, we will denote by $\rho(\mathfrak{o})$ the half sum of the roots of $\mathfrak{h}$ in $\mathfrak{o}$. Likewise, if $\mathfrak{o} \subset \mathfrak{g}$ is $\mathfrak{t}$-invariant, we will denote by $\rho(\mathfrak{o})$ the half sum of the roots of $\mathfrak{t}$ in $\mathfrak{o}$, counted with multiplicity. In this way, 
we have $\rho_{\mathfrak{g}}=\rho(\mathfrak{n}), \rho_{\mathfrak{k}}=\rho(\mathfrak{n} \cap \mathfrak{k}), \rho_{\mathfrak{l}}=\rho(\mathfrak{n} \cap \mathfrak{l})$ and $\rho_{\mathfrak{l} \cap \mathfrak{k}}=\rho(\mathfrak{n} \cap \mathfrak{l} \cap \mathfrak{k})$, but also $\rho(\mathfrak{u}), \rho(\mathfrak{u} \cap \mathfrak{k}), \rho(\mathfrak{n} \cap \mathfrak{l} \cap \mathfrak{p})$, etc.

We denote by $\langle$,$\rangle the usual W_{\mathfrak{g}}$-invariant inner product on $\mathfrak{h}^{*}$ induced by the form $B$.

THEOREM 3.1. Let $Z$ be an irreducible unitary $(\mathfrak{l}, L \cap K)$-module with infinitesimal character $\lambda$. Assume that $\lambda$ is real (i.e., in the real span of $\Delta(\mathfrak{g}, \mathfrak{h}))$, and in the good range, i.e.,

$$
\langle\lambda+\rho(\mathfrak{u}), \alpha\rangle>0, \quad \forall \alpha \in \Delta(\mathfrak{u}) .
$$

Then

1. $\mathcal{L}_{i}(Z)=\mathcal{R}^{i}(Z)=0$ for all $i \neq S$, where $S=\operatorname{dim}(\mathfrak{u} \cap \mathfrak{k})$;

2. $\mathcal{L}_{S}(Z) \cong \mathcal{R}^{S}(Z)$ is a nonzero irreducible unitary $(\mathfrak{g}, K)$-module with infinitesimal character $\lambda+\rho(\mathfrak{u})$.

For the proofs, see [KV, Chapters V, VIII and IX].

We are skipping most of the results about $K$-types of cohomologically induced modules, like upper bounds for their multiplicities and alternating formulas. We will however need the following result about some specific $K$ types, the so called bottom layer $K$-types. See [KV, V.6].

Theorem 3.2. Let $Z$ be an irreducible $(\mathfrak{l}, L \cap K)$-module. Let $\mu_{L}$ be the highest weight of an $L \cap K$-type $E\left(\mu_{L}\right)$ appearing in $Z$ with multiplicity $m$. Assume that

$$
\mu_{G}=\mu_{L}+2 \rho(\mathfrak{u} \cap \mathfrak{p}) \quad \text { is dominant for } K .
$$

Then the $K$-type $E\left(\mu_{G}\right)$ appears in $\mathcal{L}_{S}(Z)$ and $\mathcal{R}^{S}(Z)$ with multiplicity $m$.

\section{Dirac COHOMOlOgY OF COHOMOlOGiCALLY INDUCED MOdUleS}

Let $Z$ be an irreducible unitary $(\mathfrak{l}, L \cap K)$-module with real infinitesimal character $\lambda$ satisfying the condition (3.1). Assume that $Z$ has nonzero Dirac cohomology, and assume that the $L \cap K$-type $E\left(\tau_{L}\right)$ with highest weight $\tau_{L}$ contributes to $H_{D}(Z)$. By Corollary 2.2,

$$
\tau_{L}=w\left(\mu_{L}-\rho(\mathfrak{n} \cap \mathfrak{l} \cap \mathfrak{p})\right),
$$

where $\mu_{L}$ is the highest weight of an $L \cap K$-type appearing in $Z$ and $w$ is an element of $W_{\mathfrak{l} \cap \mathfrak{k}}$ making $\mu_{L}-\rho(\mathfrak{n} \cap \mathfrak{l} \cap \mathfrak{p})$ dominant for $L \cap K$. Here, if necessary, we change our choice of positive roots so that it matches the choice in Corollary 2.2.

We are going to assume that

$$
\mu_{L}-2 \rho(\mathfrak{n} \cap \mathfrak{l} \cap \mathfrak{p}) \quad \text { is dominant for } K .
$$

Then clearly $\mu_{L}-\rho(\mathfrak{n} \cap \mathfrak{l} \cap \mathfrak{p})$ is dominant for $L \cap K$, so we can take $w=1$ in (4.1), i.e.,

$$
\tau_{L}=\mu_{L}-\rho(\mathfrak{n} \cap \mathfrak{l} \cap \mathfrak{p}) .
$$


On the other hand, by Theorem 2.1, we have

$$
\tau_{L}=w_{1} \lambda-\rho_{\mathfrak{l} \cap \mathfrak{k}}, \quad \text { for some } w_{1} \in W_{\mathfrak{l}} .
$$

Using (4.3) and (4.4) we can express $\mu_{L}$ as $w_{1} \lambda-\rho_{\mathfrak{l} \cap \mathfrak{k}}+\rho(\mathfrak{n} \cap \mathfrak{l} \cap \mathfrak{p})$, and hence the weight $\mu_{G}=\mu_{L}+2 \rho(\mathfrak{u} \cap \mathfrak{p})$ defined in Theorem 3.2 is

$$
\mu_{G}=w_{1} \lambda-\rho_{\mathfrak{l} \cap \mathfrak{k}}+\rho(\mathfrak{n} \cap \mathfrak{l} \cap \mathfrak{p})+2 \rho(\mathfrak{u} \cap \mathfrak{p})=w_{1} \lambda-\rho_{\mathfrak{l}}+2 \rho(\mathfrak{n} \cap \mathfrak{p}) .
$$

Since we can write $\mu_{G}=\mu_{L}-2 \rho(\mathfrak{n} \cap \mathfrak{l} \cap \mathfrak{p})+2 \rho(\mathfrak{n} \cap \mathfrak{p}), \mu_{G}$ is $K$-dominant by the assumption (4.2), and the obvious fact that $2 \rho(\mathfrak{n} \cap \mathfrak{p})$ is $K$-dominant. It now follows from Theorem 3.2 that the $K$-type $E\left(\mu_{G}\right)$ appears in the bottom layer of $\mathcal{L}_{S}(Z) \cong \mathcal{R}^{S}(Z)$.

Furthermore, the weight $\mu_{G}-\rho(\mathfrak{n} \cap \mathfrak{p})=\mu_{L}-2 \rho(\mathfrak{n} \cap \mathfrak{l} \cap \mathfrak{p})+\rho(\mathfrak{n} \cap \mathfrak{p})$ is still $K$-dominant. It follows that

$$
\tau_{G}=\mu_{G}-\rho(\mathfrak{n} \cap \mathfrak{p})
$$

appears in $\mathcal{L}_{S}(Z) \otimes S_{\mathfrak{p}}$ as the PRV component of $E\left(\mu_{G}\right)$ tensored with the component of the spin module $S_{\mathfrak{p}}$ for $(\mathfrak{g}, \mathfrak{k})$ with lowest weight $-\rho(\mathfrak{n} \cap \mathfrak{p})$.

TheOREM 4.1. Let $Z$ be an irreducible unitary $(\mathfrak{l}, L \cap K)$-module with real infinitesimal character $\lambda$ satisfying the condition (3.1). Let $\tau_{L}, \mu_{L}, \mu_{G}$ and $\tau_{G}$ be as above. Assume that $E\left(\tau_{L}\right)$ contributes to the Dirac cohomology of $Z$, and that (4.2) holds.

Then the Dirac cohomology of the irreducible unitary (g, K)-module $\mathcal{L}_{S}(Z)$ contains the $\tilde{K}$-type $E\left(\tau_{G}\right)$. In particular, it is not zero.

Proof. We have already seen that $E\left(\tau_{G}\right)$ appears in $\mathcal{L}_{S}(Z) \otimes S_{\mathfrak{p}}$. Therefore, by Theorem 2.1, we only need to check that

$$
\tau_{G}+\rho_{\mathfrak{k}}=w(\lambda+\rho(\mathfrak{u})), \quad \text { for some } w \in W_{\mathfrak{g}} .
$$

Namely, the infinitesimal character of $\mathcal{L}_{S}(Z)$ is $\Lambda=\lambda+\rho(\mathfrak{u})$.

Using (4.6) and (4.5), we can write

$\tau_{G}+\rho_{\mathfrak{k}}=\mu_{G}-\rho(\mathfrak{n} \cap \mathfrak{p})+\rho_{\mathfrak{k}}=w_{1} \lambda-\rho_{\mathfrak{l}}+\rho(\mathfrak{n} \cap \mathfrak{p})+\rho_{\mathfrak{k}}=w_{1} \lambda-\rho_{\mathfrak{l}}+\rho_{\mathfrak{g}}=w_{1} \lambda+\rho(\mathfrak{u})$.

Therefore the following simple and well known lemma shows that we can take $w=w_{1}$ and this finishes the proof of the theorem.

Lemma 4.2. Let $\mathfrak{q}=\mathfrak{l} \oplus \mathfrak{u}$ be a $\theta$-stable parabolic subalgebra of $\mathfrak{g}$. Let $w_{1} \in W_{\mathfrak{l}}$. Then $w_{1} \rho(\mathfrak{u})=\rho(\mathfrak{u})$.

Proof. Recall that we have chosen positive roots for $\mathfrak{g}$ to consist of positive roots for $\mathfrak{l}$ and roots for $\mathfrak{u}$. The Weyl group $W_{\mathfrak{l}} \subset W_{\mathfrak{g}}$ is generated by reflections with respect to positive roots for $\mathfrak{l}$. If $\alpha$ is a positive root for $\mathfrak{l}$, then the reflection $s_{\alpha}$ sends $\alpha$ to $-\alpha$ and permutes other positive roots. In particular, $s_{\alpha}$ permutes $\Delta(\mathfrak{u})$ and hence leaves $\rho(\mathfrak{u})$ invariant. Since $w_{1}$ is a product of such $s_{\alpha}$, the lemma follows. 


\section{REFERENCES}

[C] C. Chevalley, The algebraic theory of spinors, Columbia University Press, 1954.

[EHW] T. J. Enright, R. Howe and N. R. Wallach, A classification of unitary highest weight modules, in Representation theory of reductive groups, Park City, Utah, 1982, Birkhäuser, Boston, 1983, 97-143.

[HKP] J.-S. Huang, Y.-F. Kang and P. Pandžić, Dirac cohomology of some Harish-Chandra modules, Transform. Groups 14 (2009), 163-173.

[HP1] J.-S. Huang and P. Pandžić, Dirac cohomology, unitary representations and a proof of a conjecture of Vogan, J. Amer. Math. Soc. 15 (2002), 185-202.

[HP2] J.-S. Huang and P. Pandžić, Dirac operators in representation theory, Mathematics: Theory and Applications, Birkhauser, 2006.

[HPP] J.-S. Huang, P. Pandžić and V. Protsak, Dirac cohomology of Wallach representations, preprint, 2009.

[HPR] J.-S. Huang, P. Pandžić and D. Renard, Dirac operators and Lie algebra cohomology, Represent. Theory 10 (2006), 299-313.

$[\mathrm{KV}]$ A. W. Knapp and D. A. Vogan, Cohomological induction and unitary representations, Princeton University Press, 1995.

[K1] B. Kostant, Clifford algebra analogue of the Hopf-Koszul-Samelson theorem, the $\rho$-decomposition $C(\mathfrak{g})=$ End $V_{\rho} \otimes C(P)$, and the $\mathfrak{g}$-module structure of $\wedge \mathfrak{g}$, Adv. Math. 125 (1997), no. 2, 275-350.

[K2] B. Kostant, A cubic Dirac operator and the emergence of Euler number multiplets of representations for equal rank subgroups, Duke Math. J. 100 (1999), 447-501.

[P] R. Parthasarathy, Dirac operator and the discrete series, Ann. of Math. 96 (1972), $1-30$.

[PRV] K. R. Parthasarathy, R. Ranga Rao and V. S. Varadarajan, Representations of complex semisimple Lie groups and Lie algebras, Ann. of Math. 85 (1967), 383429.

[V1] D. A. Vogan, Representations of real reductive Lie groups, Birkhäuser, Boston, 1981.

[V2] D. A. Vogan, Dirac operators and unitary representations, 3 talks at MIT Lie groups seminar, Fall 1997.

[VZ] D. A. Vogan, G. J. Zuckerman, Unitary representations with non-zero cohomology, Compositio Math. 53 (1984), 51-90.

[W] N. R. Wallach, Real Reductive Groups, Volume I, Academic Press, 1988.

\section{P. Pandžić}

Department of Mathematics

University of Zagreb

Bijenička 30, 10000 Zagreb

Croatia

E-mail: pandzic@math.hr

Received: 3.12.2009. 